\title{
A Novel Complex: A Quantum Dot Conjugated to an Active T7 RNA Polymerase
}

\author{
Mette Eriksen, ${ }^{1}$ Peter Horvath, ${ }^{2}$ Michael A. Sørensen, ${ }^{3}$ \\ Szabolcs Semsey, ${ }^{1}$ Lene B. Oddershede, ${ }^{1}$ and Liselotte Jauffred ${ }^{1}$ \\ ${ }^{1}$ Niels Bohr Institute, University of Copenhagen, 2100 Copenhagen, Denmark \\ ${ }^{2}$ Institute of Biology, Eötvös Loránd University, Budapest 1053, Hungary \\ ${ }^{3}$ Institute of Biology, University of Copenhagen, 2200 Copenhagen, Denmark \\ Correspondence should be addressed to Liselotte Jauffred; jauffred@nbi.dk
}

Received 28 January 2013; Accepted 17 April 2013

Academic Editor: Christophe Merlin

Copyright (C) 2013 Mette Eriksen et al. This is an open access article distributed under the Creative Commons Attribution License, which permits unrestricted use, distribution, and reproduction in any medium, provided the original work is properly cited.

\begin{abstract}
To perform single-molecule studies of the T7 RNA polymerase, it is crucial to visualize an individual T7 RNA polymerase, for example, through a fluorescent signal. We present a novel complex combining two different molecular functions, an active T7 RNA polymerase and a highly luminescent nanoparticle, a quantum dot. The complex has the advantage of both constituents: the complex can traffic along DNA and simultaneously be visualized, both at the ensemble and at the single-molecule level. The labeling was mediated through an in vivo biotinylation of a His-tagged T7 RNA polymerase and subsequent binding of a streptavidin-coated quantum dot. Our technique allows for easy purification of the quantum dot labeled T7 RNA polymerases from the reactants. Also, the conjugation does not alter the functionality of the polymerase; it retains the ability to bind and transcribe.
\end{abstract}

\section{Introduction}

RNA polymerase (RNAP) is an enzyme that catalyses transcription. It shall balance two tasks: binding to specific sites on DNA where transcription is initiated (promoters) and the ability to disengage at the termination signals. Proteins and other cofactors can inhibit or recruit RNAPs to DNA, thus regulating the initiation process.

Several families of RNAPs exist which differ in their molecular characteristics. The viral T7 RNA polymerase is a single subunit RNAP ( $98 \mathrm{kDa})$ [1] characterized by extreme promoter specificity [2] and a very low error rate [3]. The T7 family of RNAPs is structurally and evolutionary distinct from the multisubunit family of RNAPs. T7 RNAP allows specific high-level transcription and expression of cloned genes [4]; therefore, it is a key enzyme in the production of recombinant proteins and also in several in vitro molecular biology applications $[5,6]$. Such applications include the generation of large quantities of RNA transcripts for translation, synthesis of tRNA, rRNA, RNA virus genomes, ribozymes, microarray targets [7], production of substrates for RNA splicing [8], RNA secondary structure, antisense RNA, and RNA-protein interaction studies. The T7 RNAP is suitable for homogeneous radioactive or nonradioactive labeling of single-stranded RNA, and it plays an important role in technologies based on RNA and DNA hybridization [9]. Tight regulation of T7 RNAP expression is possible and T7 systems are therefore used for the production of otherwise deleterious protein products [10].

A wide variety of assays are designed to study the diffusive or directed translocation of molecular motors. In order to visualize or manipulate the individual motor, it is typically conjugated to a fluorescent probe or a dielectric particle. An effective conjugation, which does not alter the activity of the enzyme, is absolutely essential in order to obtain trustworthy results.

Due to their high quantum yield and large photostability [11-14] colloidal quantum dots (QDs) are a particularly good choice as labels for molecular motors. QDs have been used, for example, to track the motility of individual receptors in a cell membrane [15], to track individual bacteriophages infecting bacteria [16] and to monitor individual proteins 


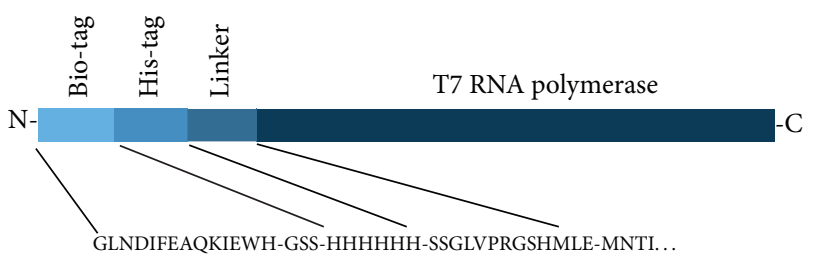

FIGURE 1: N-terminal amino acid sequence of the biotinylated T7 RNAP. The N-terminal part of the enzyme begins with a $15 \mathrm{aa}$ sequence recognized by biotin ligase. Then follows a 3 aa spacer and the His6-tag whereupon the aa sequence of the T7 RNAP begins.

diffusing on DNA $[17,18]$. Individual colloidal QDs can even be optically manipulated [19] and excited by the trapping laser through two-photon absorption [20], thus enabling both visualization and manipulation through specific attachment of a single QD. For these reasons, it is highly desirable to devise a method to specifically attach QDs to individual molecular motors. QDs can be taken up by living cells [21] and are nontoxic when encapsulated. Hence, they also have potential for in vivo studies of, for example, the action of molecular motors.

Here, we devise a technique to specifically label individual T7 RNAPs by QDs through a biotin-streptavidin bond, and we show that the QD-functionalized T7 RNAP (QD: T7RNAP) retains activity. Previously, activity was proven of a QD-labelled E. coli RNAP [22], and activity was proven of T7 RNAP bioconjugated to polystyrene beads [23]; however, to our knowledge this is the first report of QD labelling of viral T7 RNAPs that retain activity after QD marking. Moreover, our applied purification technique is simple, inexpensive, and very efficient.

\section{Materials and Methods}

2.1. In Vivo Biotinylation and Purification of T7 RNAP. Our construct was based on plasmid pBH161 which was a generous gift from William T. McAllister, Department of Biochemistry, University of New Hampshire. Plasmid pBH161 encodes a T7 RNAP His-tagged $\mathrm{N}$ terminally [24]. We further tagged the enzyme $\mathrm{N}$ terminally with the GLNDIFEAQKIEWH sequence, referred to as a biotag, which was recognized by biotin ligase and biotinylated at a single lysine in vivo [25] (Figure 1). The DNA sequence encoding the Bio-tag was synthesized by PCR using three overlapping primers:

\section{5'-ATCACCATGGGTAGCAGCGGCCTGAAC- GACATCTTCGAAGCTCAGAAAA- $3^{\prime}$. \\ $5^{\prime}$-ATCTTCGAAGCTCAGAAAATTGAATGG- CACGGCAGCTCTCATCATCATCATCATCAT- AG-3'. \\ $5^{\prime}$-ATAGCTCGAGCATATGAGAACCGCGCG- GCACCAGACCACTGCTATGATGATGATGAT- GATGAG-3'.}

The synthesized DNA fragment was digested by XhoI and $\mathrm{NcoI}$ and inserted between the XhoI and NcoI sites in pBH161 upstream of the sequence encoding the His-tag; the resulting plasmid was denoted as pBioT7. Bio-His-tagged T7 RNAP (Bio-T7 RNAP) was expressed in the E. coli strain BL21Star (Invitrogen) containing the plasmids: pBioT7 and pBirA (Avidity), which expresses biotin-holoenzyme-synthetase activities. Enzymes were purified from the lysate using $\mathrm{Ni}^{2+}$ agarose beads (Qiagen) and eluted with $80 \mathrm{mM}$ imidazol.

2.2. Quantification of Biotinylation of T7 RNAP. To quantify the amount of Bio-T7 RNAPs, purified Bio-T7 RNAPs were mixed with SoftLink Soft Release Avidin Resin (Promega) and allowed to bind for 1 hour at room temperature. The mixture was loaded on Poly-Prep Chromatography Columns (Bio-Rad) and the flow-through was collected. The column was washed with 10 bed volumes of buffer $(20 \mathrm{mM}$ Tris$\mathrm{HCl} \mathrm{pH} 7.9,0.5 \mathrm{M} \mathrm{NaCl}, 0.05 \%$ (w/v) Tween 20). Samples of the flow-through, the washed fraction, and the matrixbound fractions were loaded on a $4-12 \%$ NuPAGE gel (Invitrogen) along with a molecular weight marker (SeeBlue Plus2, Invitrogen). The control protein (Phage 16-3 repressor, C16-3) was not biotinylated. After electrophoresis the gel was stained with Coomassie Brilliant Blue.

2.3. Estimation of Concentration of Biotinylated T7 RNAPs. We used the ExPASy ProtParam tool (expasy.org) [26] to calculate the theoretical extinction coefficient of the BioT7 RNAP to $1457601 / \mathrm{M} * \mathrm{~cm}$. The absorbance was measured using a NanoDrop spectrophotometer (ThermoScientific). The enzyme preparation also included impurities (Figure S1, see supplementary material available online at http://dx.doi .org/10.1155/2013/468105) of unknown extinction coefficient. The approximate enzyme concentration was estimated using the measured absorbance and Beer-Lamberts equation.

2.4. Verification of Activity of Biotinylated T7 RNAPs. The in vivo biotinylated T7 RNAPs were immersed in a reaction mixture containing $2 \mathrm{ng} / \mu \mathrm{L} 1,000 \mathrm{bp}$ DNA template with T7 promoter and terminator, transcription buffer $(40 \mathrm{mM}$ Tris- $\mathrm{HCl} \mathrm{pH} \mathrm{7.9,} 6 \mathrm{mM} \mathrm{MgCl}_{2}$, and $10 \mathrm{mM} \mathrm{NaCl}$ ), $2 \mathrm{mM}$ spermidine, $10 \mathrm{mM}$ DTT, $2 \mathrm{mM}$ of each rNTP, 2 units $/ \mu \mathrm{L}$ RNasin, and $2.6 \mu \mathrm{M}$ Bio-T7 RNAP. The reaction mixture was incubated at $37^{\circ} \mathrm{C}$ for 1 hour and loaded onto a $1.8 \%$ agarose gel and stained with ethidium bromide.

2.5. Formation of $\mathrm{QD}: \mathrm{T} 7 \mathrm{RNAP}$ Complexes. The Bio-T7 RNAPs were attached to QDs in the following manner. In a final volume of $30 \mu \mathrm{L} 0.13,0.091$, or $0.052 \mathrm{nmol}$ of the Bio-T7 RNAP were mixed with $10 \mathrm{mM} \mathrm{DTT}$, transcription buffer, and $0.01 \mathrm{nmol}$ streptavidin-coated QDs (Qdot605, Invitrogen). The mixture was left at $4^{\circ} \mathrm{C}$ for 1.5 hour allowing the streptavidin-biotin bonds to form. Parallel controls consisted of the same solution with Bio-T7 RNAPs but without QDs present.

2.6. Purification of $Q D$ : T7RNAP Complexes. In order to purify the QD: T7RNAP complexes $20 \mu \mathrm{L}$ transcription buffer was added to the QD : T7RNAP and control solutions. Each of the final $50 \mu \mathrm{L}$ reactions was transferred to $25 \mu \mathrm{L}$ avidin 


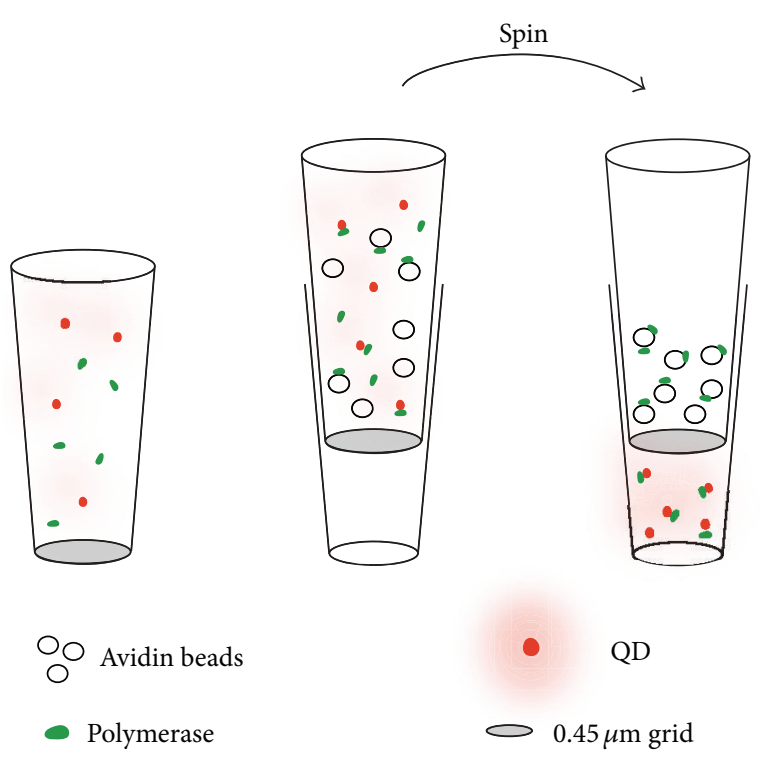

FIGURE 2: Separation of QD : T7RNAP complexes from free RNAPs. The Bio-T7 RNAPs are mixed with streptavidin-coated QDs thus forming QD : T7RNAP complexes. The suspension is mixed with an avidin resin to capture the remaining free Bio-T7 RNAPs. Once the suspension is centrifuged on a $0.45 \mu \mathrm{m}$ filter only the QD : T7RNAP complexes and free QDs reach the bottom.

resin, prewashed and centrifuged. The resin was resuspended, and put on a shaker at $4^{\circ} \mathrm{C}$ for 1 hour. The avidin and QD : T7RNAP suspensions were filter-centrifuged (pore size $0.45 \mu \mathrm{m}$, Pierce), only allowing the QD:T7RNAP and the free QDs to be in the flow-through, which was collected for an activity test (Figure 2). The avidin resins were washed with $100 \mu \mathrm{L}$ transcription buffer and resuspended in $10 \mu \mathrm{L}$ transcription buffer and also collected for an activity test.

2.7. Activity of QD:T7RNAP Complexes and Avidin-Bound RNAPs. A 1,000 bp T7 RNAP transcription template was made by PCR on pJT512 (the 11/6 construct of Tholstrup et al.2012 [27]). Forward primer was TH414:

\section{5' -TAATACGACTCACTATAGGGAGAGTATACC- TCTCAGTTGGGTG-3}

(underlined is overhang containing T7 promotor) and the reverse primer, TH412, had the sequence:

\section{5'-ATAATTCGCGTCTGGCCTTC.}

This template supports a $1000 \mathrm{nt}$ long transcript from the promoter to the end of the fragment. All activity tests were made with this template, except in Figures 4(b) and 4(c) where the template pMAS30 (plasmid encoding upstream lacZ riboprobe [27]), digested with SacI, was used. This T7 RNAP template supports a $830 \mathrm{nt}$ long transcript.

Activity tests were performed of both the flow-through containing QD:T7RNAP complexes and of the washed avidin resin containing Bio-T7 RNAPs bound to the avidin resin. The reaction mixture $(10 \mu \mathrm{L})$ contained $2 \mathrm{ng} / \mu \mathrm{L}$, 1,000 bp DNA template with a T7 promoter, transcription buffer, $2 \mathrm{mM}$ spermidine, $10 \mathrm{mM}$ DTT, $2 \mathrm{mM}$ of each rNTP, 2 units/ $\mu \mathrm{L}$ RNasin, and $6 \mu \mathrm{L}$ of collected QD : T7RNAP solution. The reaction mixture was incubated at $37^{\circ} \mathrm{C}$ for 1 hour and loaded on a $1.8 \%$ agarose gel. The two controls consisted of $0.026 \mathrm{nmol}$ Bio-T7 RNAPs in transcription buffer and 1 pmol QDs in transcription buffer.

As each QD is coated with 6-8 streptavidins (Invitrogen), the average number of Bio-T7 RNAPs bound to each QD can be controlled by adjusting Bio-T7 RNAP and QD concentrations. The RNA product of the activity test should not derive from free Bio-T7 RNAPs. In order to find the amount of RNA product originating from free BioT7 RNAPs, controls undergoing the same procedure as the samples, but with no QDs, revealed the amount of free Bio-T7 RNAPs escaping the avidin resin in the flow-through. In this way the concentrations of Bio-T7 RNAPs and QDs yielding optimal working conditions, that is, an absolute minimum of free Bio-T7 RNAPs, were found.

2.8. T7 RNAP Streptavidin Control. A gel filtration was performed in order to exclude the possibility that active QD : T7RNAPs were only attached to streptavidin (and not to a QD). In this experiment $0.385 \mathrm{nmol}$ Bio-T7 RNAP was mixed with $0.075 \mathrm{nmol}$ QDs (Bio-T7 RNAP: $\mathrm{QD} \simeq 5: 1$ ) in a final volume of $110 \mu \mathrm{L}$, and the solution was left at $4^{\circ} \mathrm{C}$ for 1.5 hour to allow the formation of complexes. The solution was filtered through a Superose 6 10/300 GL column on BioRad BioLogic DuoFlow at a flow rate of $0.5 \mathrm{~mL} /$ minute transcription buffer. $0.5 \mathrm{~mL}$ fractions were collected with BioRad BioLogic BioFrac Fraction Collector. Controls without QDs were processed similarly using $2.137 \mathrm{nmol}$ and $0.385 \mathrm{nmol}$ Bio-T7 RNAP, respectively.

An activity test was performed on the gel filtration fractions. To this end $1 / 50(10 \mu \mathrm{L})$ of each collected fraction was supplemented to a final volume of $12.1 \mu \mathrm{L}$ with spermidine, DTT, rNTPs, and RNAse inhibitor (at concentrations described for the previous activity tests) and $20 \mathrm{ng}$ DNA. The samples were incubated at $37^{\circ} \mathrm{C}$ for 2.5 hours before electrophoresis on $1.8 \%$ agarose gels. The $2.137 \mathrm{nmol}$ Bio-T7 RNAP control was tested with $\sim 115$ ng PCR-amplified DNA template from pJT512.

2.9. Single Molecule Assay. A 3,250 bp piece of $\lambda$-DNA was synthesized by PCR using the primers:

\section{5 '-TTTTGAATTCAAAAAACCCCTCAAGAC- CCGTTTAGAGGCCCCAAGGGGTTCTCATGC- TGAAAACGTGGTGTACCGGC-3'; \\ $5^{\prime}$-TTTTTTAAGCTTGGACCTATCTGCCCG- TTCGTCCCGTCGTT-3'.}

This DNA piece was inserted between the EcoRI and HindIII of the plasmid pGEM4Z (Promega) downstream of the T7 promoter. Using this plasmid pLJP1 as a template, a $\sim 3,000$ bp DNA was constructed with biotin and digoxigenin labels at the ends using the following primers:

5'-Bio-TGTGATGCTCGTCAGGGGGGOGGAGC-3' $5^{\prime}$-Dig-TTCTCATGCTGAAAACGTGGTGTACCGGC-3'. 


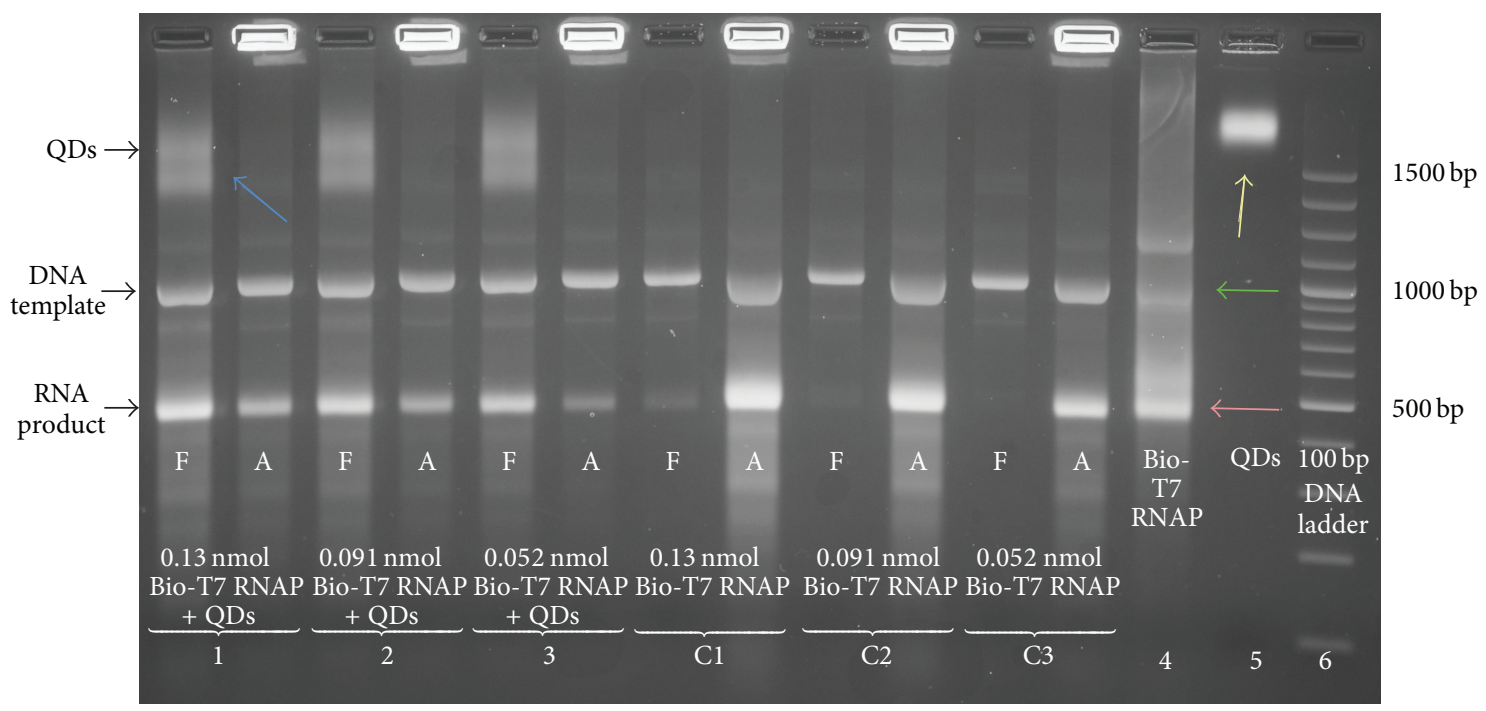

FIGURE 3: Activity test of purified QD : T7RNAP complexes. Lanes 1-3 and C1-C3 show the activity of $6 \mu \mathrm{L}$ of the flow-through (F) and of the washed avidin resin (A). In lane 1 a total amount of $0.13 \mathrm{nmol}$ Bio-T7 RNAP was used, in lane $20.091 \mathrm{nmol}$, and in lane $30.052 \mathrm{nmol}$. In all lanes 1-3 the amount of QDs was $0.01 \mathrm{nmol}$. C1, C2, and C3 are parallel controls of lanes 1, 2, and 3, respectively, using the same amount of Bio-T7 RNAP but without QDs. Lane 4 shows the activity of $0.026 \mathrm{nmol}$ free Bio-T7 RNAP, lane 51 pmol QDs alone, and lane 6 is a $100 \mathrm{bp}$ DNA ladder. The red arrow marks the RNA product, green arrow marks DNA template, blue arrow marks QD : T7RNAP bands, and yellow arrow marks bands of free QDs. The material lighting up in the slots in the A lanes is the avidin resin unable to move into the gel, compared, for example, to lane 2A in Figure S3.

The DNA product was purified with QIAquick Gel Extraction Kit. At $\sim 500$ bp from the biotinylated end, the DNA construct had a T7 promoter. There was no terminator site present.

A perfusion chamber was made by placing parafilm forming a $20-25 \mu \mathrm{L}$ well on a $60 \times 24 \mathrm{~mm}$ glass slide and adding a $50 \times 24 \mathrm{~mm}$ glass slide as lid. The chamber was heated such that the parafilm melted to the glass surfaces. To coat the surface with antidigoxigenin $20 \mu \mathrm{g} / \mathrm{mL}$ antidigoxigenin in TE (10 mM Tris-HCl (pH 8), 1 mM EDTA) was flushed into the chamber and left at $4^{\circ} \mathrm{C}$ overnight or at least 1 hour at $25^{\circ} \mathrm{C}$. To minimize unspecific bindings to the glass surface transcription buffer containing $2 \mathrm{mg} / \mathrm{mL} \alpha$-casein was flushed through the chamber and incubated for 1 hour at $25^{\circ} \mathrm{C} .10 \mathrm{nM}$ of the DNA solution was flushed into the chamber and incubated for 30 minutes at $25^{\circ} \mathrm{C}$ such that the digoxigenin-labeled end of the DNA tether could bind to the antidigoxigenin-coated surface. $2 \mathrm{mg} / \mathrm{mL} \alpha$-casein in transcription buffer was flushed into the chamber and left at $25^{\circ} \mathrm{C}$ for 30 minutes to block unspecific bindings. Finally, QD : T7RNAP complexes (2.6 nM Bio-T7 RNAP and $1 \mathrm{nM}$ QDs) in transcription buffer with $2 \mathrm{mg} / \mathrm{mL} \mathrm{BSA}, 2 \mathrm{mg} / \mathrm{mL}$ spermidine, $2 \mathrm{mM}$ of each ribonucleotides, and $10 \mathrm{mM}$ DTT were flushed into the chamber, and the chamber was sealed with vacuum grease. The controls were processed similarly but without ribonucleotides in the transcription buffer.

The samples were investigated using an inverted Leica microscope. A mercury lamp was focused with an oil immersion objective (Leica HCX PL APO $100 \times \mathrm{NA}=1.4$ oil, $\infty$ CS). To image the light emitted from the $605 \mathrm{~nm}$ QD we used a custom made filter cube (Leica HQ420/40x + 1064, z440/1065rpc, and HQ605/40m-2p) which allowed the
$420 \mathrm{~nm}$ light from the mercury lamp to be reflected up to the sample and only allowed the emitted $605 \mathrm{~nm}$ light to be transmitted to the EMCCD camera (Ixon, Andor). The images were analyzed with ImageJ and custom made Matlab routines. In addition, we performed centroid tracking of the tethered QDs using the ImageJ tracking routine Spottracker2D [28].

\section{Results and Discussion}

3.1. In Vivo Biotinylation. The T7 RNAPs were biotinylated in vivo with a procedure that parallels the method used for biotinylating a lambda phage subsequently labeled by a QD [29]. The biotinylated T7 RNAPs were purified using an agarose bead assay. The amount of biotinylated T7 RNAPs was quantified using an avidin-coated resin and estimated to be $95-99 \%$ of the total (Figure S1).

After in vivo biotinylation of T7 RNAPs we tested that they were still active. The activity was verified by inspecting the strong band at the expected weight of the product in lane 4 (Figure 3, red arrow). As native T7 RNAP did not bind the avidin resin, seen by comparing lane $3(\mathrm{~F})$ to lane 3(A) (Figure S3), the conjugation of the avidin resin and Bio-T7 RNAPs is mediated by the biotin-streptavidin bond only. The QD:T7RNAP complexes were formed by mixing the Bio-T7 RNAPs with streptavidin-coated QDs. In order to purify the QD : T7RNAP complexes they were mixed with an avidin resin, with the goal of capturing possible BioT7 RNAPs which did not bind to QDs. The suspension was filter centrifuged using a $0.45 \mu \mathrm{m}$ filter (Figure 2) and the QD:T7RNAP complexes would be present in the flowthrough. Free QDs would also pass the filter and be present 


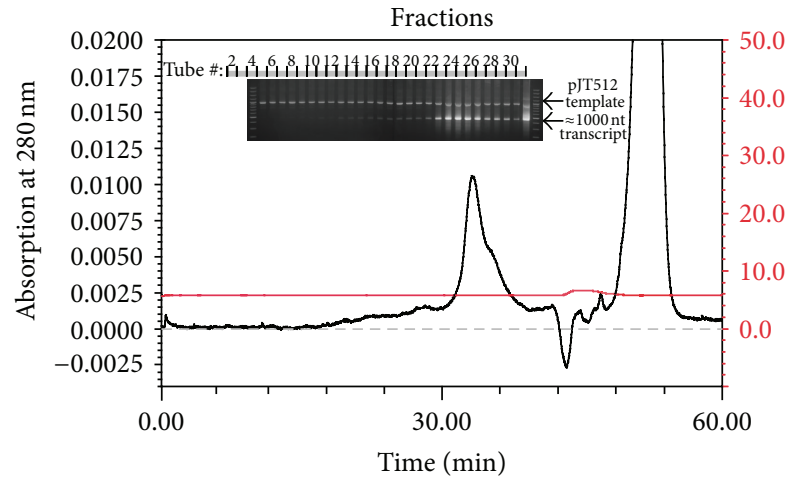

(a)

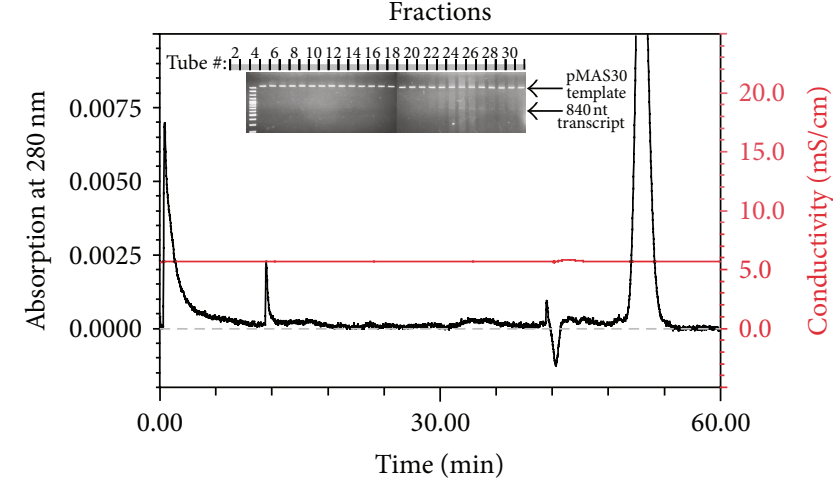

(b)

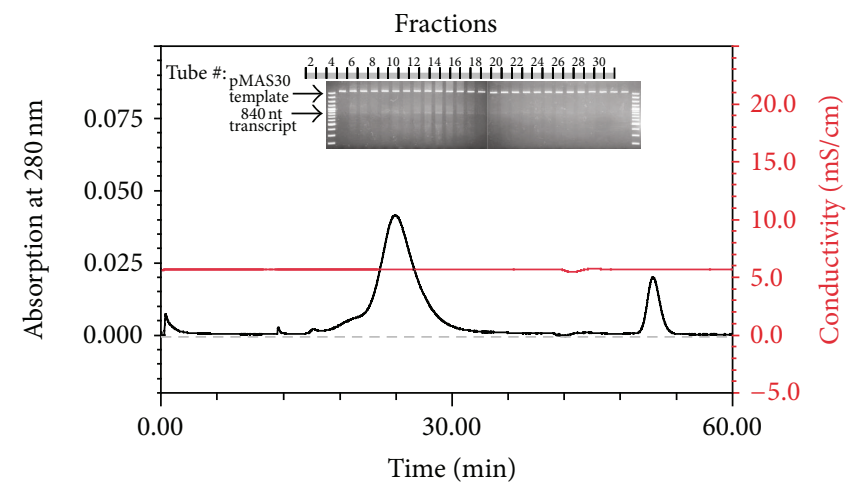

(c)

Figure 4: Gel filtration of QD:T7RNAP complexes. This experiment was performed to exclude the possibility that the active Bio-T7 RNAP was bound to streptavidin (and not to QDs). (a) Control without QDs using 2.137 nmol Bio-T7 RNAP, (b) control without QDs using $0.385 \mathrm{nmol}$ Bio-T7 RNAP, and (c) $0.385 \mathrm{nmol}$ Bio-T7 RNAP + $0.075 \mathrm{nmol}$ QDs (Bio-T7RNAP: QD $\simeq 5: 1$ ). All three graphs show the absorption of $280 \mathrm{~nm}$ light as function of time (left axis) as well as the conductivity (right axis). In all three experiments the sample was filtered through a Superose $610 / 300 \mathrm{GL}$ column at a flow rate of $0.5 \mathrm{~mL} /$ minute, and fractions of $0.5 \mathrm{~mL}$ were collected from 11 to $40 \mathrm{minutes}$. In each of panels (a), (b), and (c) an inserted gel shows the activity found in the individual fractions. Fraction 32 of panel a is a positive control of Bio-T7 RNAP activity. The DNA MW marker used (left lane in all three inserted gels) was from top $3 \mathrm{kbp}, 2 \mathrm{kbp}, 1.5 \mathrm{kbp}, 1.2 \mathrm{kbp}, 1 \mathrm{kbp}, 900 \mathrm{bp}$ $800 \mathrm{bp}, 700 \mathrm{bp}, 600 \mathrm{bp}$, and so forth. Equal amounts of the DNA MW marker were loaded on each gel. Bio-T7 RNAP (103 kDa) was eluted at 33.2 minutes (fraction 24) and QDs at 24 minutes (fraction 15). In a (not shown) calibration E. coli GlyS (224 kDa) eluted at 28.4 minutes, Dextran blue at 14.5 minutes (fraction 5), and $70 \mathrm{~S}$ ribosomes $(\sim 3000 \mathrm{kDa})$ at 20.8 minutes (fraction 13$)$.

in the flow-through as well as possible streptavidin:T7RNAPs released from the QDs. There has been earlier attempts to tag T7 RNAPs [18], but the activity of the protein, when conjugated to a QD, was not confirmed.

3.2. Activity of Ensembles of $Q D: T 7 R N A P$ Complexes. The activities of the purified QD:T7RNAP complexes and the corresponding controls without QDs were investigated using normal gel electrophoresis (Figure 3 ). The RNA products (red arrow) from the flow-through (F) and avidin resin (A) are shown for Bio-T7 RNAP concentrations in the initial binding mixture of, respectively, $0.13,0.091$, and $0.052 \mathrm{nmol}$ in lanes marked 1, 2, and 3 and QD concentrations of $0.01 \mathrm{nmol}$. This corresponds to QD:T7RNAP ratios of about 1:13, 1:9 and $1: 5$, respectively. The corresponding controls, without QDs, are shown in lanes marked $\mathrm{C} 1, \mathrm{C} 2$, and $\mathrm{C} 3$. There is a clear band denoting the existence of an RNA product in all lanes originating from QD : T7RNAP complexes and in all control lanes, albeit, only in the avidin resin (A) of the latter. This verifies that the RNA product present, for example, in lane
3 (F) originated from QD : T7RNAP complexes only since no RNA product is present in the flow-through of C3. This also shows that the surplus of free Bio-T7 RNAPs is efficiently captured by the avidin resin. Hence, we conclude that the BioT7 RNAP is indeed active when bound to a QD.

It is clear from lanes 1, 2, and 3 that the majority of the QDs passed the $0.45 \mu \mathrm{m}$ filter and showed up on the gel in the F columns as two distinct bands (blue arrow) at 1500-1700 bp as earlier reported [30]. Interestingly, the QDs with Bio-T7 RNAPs attached migrate further than the QD alone; to see this compare lane 5 (yellow arrow) to lanes 1, 2, or 3 (F) (blue arrow). This is probably because the net charge of the Bio-T7 RNAP at $\mathrm{pH} 8.5$ ( $\mathrm{pH}$ of the agarose gel buffer) is -5 , and therefore the QD:T7RNAP complex migrates faster towards the anode during electrophoresis than the free QDs. The agarose gel pore size was relatively large in comparison to the size of the approximately spherical QD: T7RNAP complex. Hence, the fact that one or possibly more charged Bio-T7 RNAPs are attached and thereby cause the complex to move faster in 
the field has a larger influence on the overall migration than the incremental increase in effective diameter [31].

QDs are only present in the flow-through (F) of lanes 1, 2 , and 3 (Figure 3), confirming that the majority of the QDs passed the filter and did not stick to the avidin resin. On the basis of the intensities of the bands originating from the RNA products of $\mathrm{F}$ and A we estimated a ratio 3-4:1 of RNAPs to QDs. This ratio is in accordance with a recent report on biotin binding to the same type of QDs [32].

The total amount of RNA product in the presence of QDs, lane 3, corresponds well with the RNA product in the control without QDs, lane C3 (same initial Bio-T7 RNAP concentrations). The intensities of the bands with RNA product (red arrow) in lane $3(\mathrm{~F}+\mathrm{A})$ were quantified (ImageJ, Gel Analyser) and compared to the band with RNA product (red arrow) in C3. The two samples differed in intensity by $~ 5 \%$ only; hence, the Bio-T7 RNAP retained similar activity whether bound to QDs or to the avidin resin. The fact that the Bio-T7 RNAP also binds very well to avidincoated agarose beads (Figure 3 lanes $\mathrm{Cl}-\mathrm{C} 3$ ) while retaining enzymatic activity proves the generality of the method; the Bio-T7 RNAPs retain activity though bound to other types of avidin-coated handles than a QD.

To estimate the activity loss due to QD conjugation we first determined if our activity assay responded to different polymerase concentrations (Figure S2). Next, we compared the activity of the QD:T7RNAP complex to the nonconjugated Bio-T7 RNAP (lane $1(\mathrm{~F}+\mathrm{A})$ to lane 4 in Figure S3) and corrected for dilutions of Bio-T7 RNAP in lanes 1 to a concentration of approximately $0.013 \mathrm{nmol}$. This comparison showed that the QD:T7RNAP complex lost at most $\sim 15 \%$ of its activity in the experiment shown in Figure S3. However, upon repeating the experiment 21 times with an independently purified protein batch, we found an average activity loss of $(42 \pm 15) \%$ (mean \pm STD) upon QD conjugation. Our activity test of QD-bound RNAPs is an indirect method of measuring the purity of complexes compared to gel purification [33]. However, running the complexes through an avidin resin is still reliable, time saving and requires very little preparation.

3.3. Gel Filtration of $Q D$ : T7RNAP Complexes. To exclude the possibility that the active Bio-T7 RNAP was only bound to streptavidin (and not to QDs) released from the stock solution of streptavidin-coated QDs, we mixed Bio-T7 RNAP and QDs in a molar ratio of 5:1 and performed a gel filtration on a Superose 6 column (Figure 4). This type of column facilitates the separation of high molecular weight substances and molecules in the particle size range relevant for ribosomes and QDs. The goal of this experiment is to compare the Bio-T7 RNAP activity in the fractions where QDs eluted to the activity in the fractions where streptavidin:Bio-T7 RNAP is expected to elute. In the experiment three different gel filtrations were performed, two controls without QDs with different Bio-T7 RNAP concentrations and one with QDs present. The two controls without QDs were performed using $2.137 \mathrm{nmol}$ Bio-T7 RNAP (Figure 4(a)) and $0.385 \mathrm{nmol}$ BioT7 RNAPs (Figure 4(b)), respectively. The Bio-T7 RNAPs elute at 37 minutes (fraction 25) clearly visible in Figure 4(a) and just visible in Figure 4(b). Figure 4(a) shows where free Bio-T7 RNAP elute, while Figure 4(b) is a control to Figure 4(c), which shows the signal that can be obtained with this low amount of Bio-T7 RNAP. Another important reason to use a low concentration of QDs for the gel filtration was that these experiments required a very large amount of QDs $(\sim 75 \mu \mathrm{L})$ in comparison to the other experiments described above (which each used $10 \mu \mathrm{L}$ ). As QDs are still rather expensive, it makes sense to perform the experiment with as small an amount as possible (as done in the experiments depicted in Figures 4(b) and 4(c)). It is unlikely that the streptavidin tetramer would be able to bind more than two molecules of biotin-labelled T7 RNAP due to steric hindrance $[34,35]$, and the complex would maximally obtain a molecular weight of $60 \mathrm{kDa}$ (Streptavidin) $+2 \times 103 \mathrm{kDa}$ $(\mathrm{T} 7 \mathrm{RNAP})=266 \mathrm{kDa}$. Such a complex should therefore elute approximately at 28 minutes (fraction 19), much later than the observed QD : T7RNAP activity that peaked in fractions 14-15 (inset of Figure 4(c)). The Bio-T7 RNAP peak is not visible and the corresponding activity in fraction 25 was very low compared to the early fractions. The peak at 50 minutes is caused by oxidized DTT from the storage buffer. Due to a relatively large spread in QD sizes (Figure S4) the BioT7 RNAP activity peak actually extended up to fraction 5 where the largest QDs appeared. Hence, we conclude that the activity observed was indeed from QD: T7RNAP. These results also exclude the possibility that the activities seen in our other assays originate from residual T7RNAP unbound to QDs. Because we see the same overall amount of RNA in fraction 6-17 of Figure 4(c) as is fraction 22-28 of Figure 4(b), we conclude that the activity of the complex is comparable to the activity of free Bio-T7 RNAP.

3.4. Activity of Single Molecule QD: T7RNAP Complexes. As the QD:T7RNAP complex has a large potential for singlemolecule investigations, we also tested its activity in a typical single-molecule assay, the tethered particle assay. In this assay a double-stranded DNA tether ( 3,000 bp) was specifically anchored to a surface with the free biotinylated end bound to a reporter particle, a streptavidin-coated QD. The DNA tether had a T7 promoter sequence $500 \mathrm{bp}$ from the free end, and this promoter was directed towards the glass surface. The experiment and a control were conducted as sketched in Figures 5(b) and 5(c), respectively. Intensity images of DNA tethers on the coverslip surface (Figures 5(d) and 5(e)) were collected with wide-field fluorescence microscopy and a sensitive camera.

Prior to every experiment we located the DNA tethers with the reporter QD and tracked the diffusive behavior through time to find the subpixel coordinates. The magnitude of the tethered particle motion contains information on the overall length of the tether. Therefore, the diffusion was used to verify that the observed QDs were indeed attached to a DNA tether of $\sim 1 \mu \mathrm{m}$ length and not, for example, an agglomerate of $\alpha$-casein which, most likely, has a shorter tethering length. To this end we measured the root-meansquare displacement:

$$
\rho=\sqrt{l_{1}^{2}+l_{2}^{2}}, \quad \text { where } l_{j}^{2}=\frac{1}{n} \sum_{i=1}^{n}\left(x_{j, i}-\bar{x}_{j}\right)^{2} .
$$




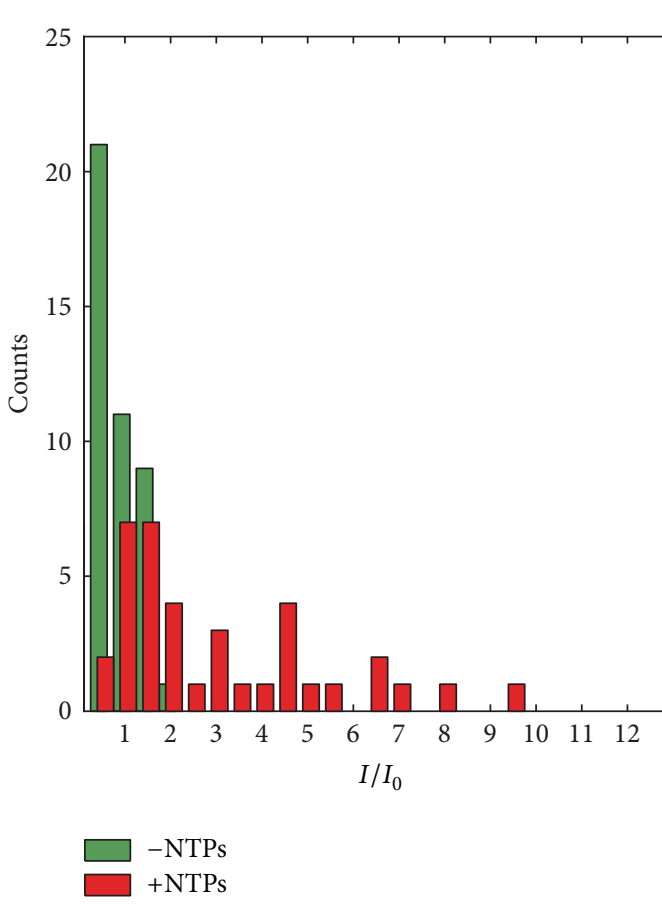

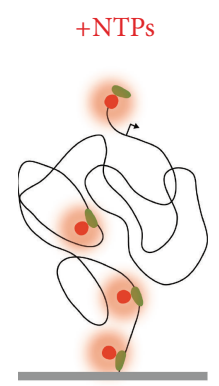

(b)

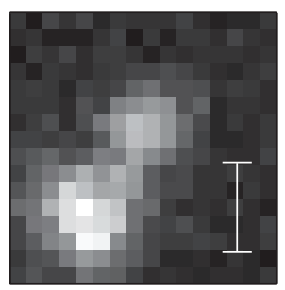

(d)

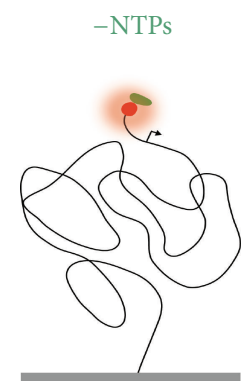

(c)

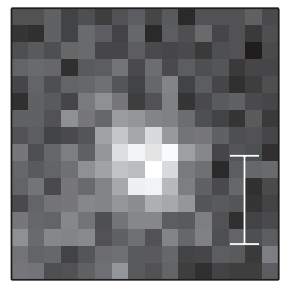

(e)

(a)

FIGURE 5: Activity of QD : T7RNAP complexes tested in a single molecule assay. In the single molecule assay the DNA tether was biotinylated at the free end, thus allowing a streptavidin-coated QD to attach to this end. In the control no ribonucleotides (NTPs) were present. Hence, as the Bio-T7 RNAP requires the initiating nucleotide (GTP) for specific binding to the promoter, it is likely that the QD at the end of the tether is the only QD affiliated with the DNA tether of the control. (a) Intensity distributions of DNA tethers with varying number of QDs attached. Red bars denote an experiment with NTP present $(n=39)$, and green bars denote the control without NTP $(n=49)$. The concentration of Bio-T7 RNAPs was $200 \mathrm{nM}$ in both cases. (b) Sketch of the +NTP experiment where QDs are attached both at the biotinylated end of the tether and as QD: T7RNAP complexes elongating along the tether. (c) Sketch of the -NTP control, only a single QD is attached at the biotinylated $5^{\prime}$ end of the tether. (d) Image of a typical +NTP experiment where QDs are actually seen at different locations along the tether. (e) Image of a typical -NTP control, only a single QD is visible. Scale bars in (d) and (e) are $500 \mathrm{~nm}$.

Here, $x_{j}, j \in\{1,2\}$, is the position of one of the two orthogonal dimensions of the projected surface plane, $\bar{x}_{j}$ is the mean position, and $n$ is the number of data points in the time series. The root-mean-square displacement of QDs attached to the free end of the DNA tether was measured: $\rho_{-\mathrm{NTP}}=$ $233 \mathrm{~nm} \pm 56 \mathrm{~nm}$ (mean \pm STD) $(N=30)$. The result is in accordance with the one reported for a QD attached to a DNA tether of similar size [36].

For the actual experiment QD : T7RNAP complexes were added to the sample together with the required ribonucleotides (NTPs). Individual QD: T7RNAP complexes thus attached at the promoter site and started transcription along the DNA tether. As there was no transcription termination sequence, the QD : T7RNAP complexes were prone to accumulate on the DNA tether close to the glass surface. In the control no NTPs were present $(-\mathrm{NTP})$; hence, it was unlikely that any QD:T7RNAP complexes would attach to the promoter or start elongating. The only fluorescent signal expected from the control was that originating from a streptavidin-coated QD attached to the biotinylated end of the DNA tether (Figure 5(e)).

In the experiments with the elongating QD:T7RNAP complexes $(+\mathrm{NTP})$ the spots on the surface were significantly more intense than in the control experiment (-NTP). Probably because the luminescent spots in the experiments originated from a single DNA tether with multiple QDs attached, only one QD would attach to a DNA tether in the control experiments. As the DNA tether itself performed thermal fluctuations in and out of the focal volume and the QD also exhibited blinking, the resulting intensity time series (with total length $\sim 200 \mathrm{~s}$ ) had significant intensity fluctuations. In the analysis we used the window of 20 frames $(\sim 5 s)$ of each time series around the maximum intensity. The average intensity, $I$, of the window was normalized to the intensity average of a time series originating from the control ( $-\mathrm{NTP}), I_{0}$. The measured values of $I / I_{0}$ both for the experiment (red bars) and for the control (green bars) are shown in Figure 5(a). The intensity distribution of the control is very narrow and peaks around a value of 1 , consistent with the expectation that only a single QD would attach to the tether. The intensity distribution of the experiment (+NTP) is significantly broader, scattered, and consistent with an average accumulation of 5 QDs per tether (at a concentration of $200 \mathrm{nM}$ Bio-T7 RNAPs). Hence, we conclude that the individual QD : T7RNAP complexes are indeed active; in the presence of NTP they bind to the promoter and accumulate on the DNA tether. 


\section{Conclusions}

We showed that the viral T7 RNAP could easily be efficiently biotinylated while retaining activity. In addition, we showed that upon attachment of a streptavidin-coated QD the QD : T7RNAP complex still retains activity. In particular, we showed how to purify QD:T7RNAPs from a solution of unbound RNAPs. As the conjugation and purification assays presented in this study were simple and highly efficient, the devised technique is recommendable for attachment and purification of QDs to Bio-T7 RNAPs. In comparison to the E. coli RNAP the viral T7 RNAP is simple as it only consists of a single polypeptide chain and has a short promoter recognition sequence. The simplicity of the T7 RNAP as well as its efficiency has paved the way for high production of specific protein products [6]. Anyhow, only few singlemolecule investigations of the T7 RNAP exist [37-40] in comparison to the detailed knowledge obtained on the single molecule level concerning the fundamental properties, for example, transcription velocity, stalling force, pausing events, backtracking, of E. coli RNAP. With the techniques here presented it will be possible to uncover the action of T7 RNAP even at the single-molecule level.

\section{Acknowledgments}

The authors acknowledge support from Jesper Tholstrup and thank Kaj Frank Jensen for introducing them to gel filtration. The work was financed through the University of Copenhagen Excellence Program.

\section{References}

[1] S. J. Stahl and K. Zinn, "Nucleotide sequence of the cloned gene for bacteriophage T7 RNA polymerase," Journal of Molecular Biology, vol. 148, no. 4, pp. 481-485, 1981.

[2] J. E. Brown, J. F. Klement, and W. T. McAllister, "Sequences of three promoters for the bacteriophage SP6 RNA polymerase," Nucleic Acids Research, vol. 14, pp. 3521-3526, 1986.

[3] S. Brakmann and S. Grzeszik, "An error-prone T7 RNA polymerase mutant generated by directed evolution," ChemBioChem, vol. 2, no. 3, pp. 212-219, 2001.

[4] F. W. Studier and B. A. Moffatt, "Use of bacteriophage T7 RNA polymerase to direct selective high-level expression of cloned genes," Journal of Molecular Biology, vol. 189, no. 1, pp. 113-130, 1986.

[5] K. Terpe, "Overview of bacterial expression systems for heterologous protein production: from molecular and biochemical fundamentals to commercial systems," Applied Microbiology and Biotechnology, vol. 72, no. 2, pp. 211-222, 2006.

[6] M. B. Iskakova, W. Szaflarski, M. Dreyfus, J. Remme, and K. H. Nierhaus, "Troubleshooting coupled in vitro transcriptiontranslation system derived from Escherichia coli cells: synthesis of high-yield fully active proteins," Nucleic Acids Research, vol. 34, no. 19, article no. e135, 2006.

[7] D. J. Lockhart, H. Dong, M. C. Byrne et al., "Expression monitoring by hybridization to high-density oligonucleotide arrays," Nature Biotechnology, vol. 14, no. 13, pp. 1675-1680, 1996.

[8] A. R. Krainer, T. Maniatis, B. Ruskin, and M. R. Green, "Normal and mutant human $\beta$-globin pre-mRNAs are faithfully and efficiently spliced in vitro," Cell, vol. 36, no. 4, pp. 993-1005, 1984.

[9] K. Zinn, D. DiMaio, and T. Maniatis, "Identification of two distinct regulatory regions adjacent to the human $\beta$-interferon gene," Cell, vol. 34, no. 3, pp. 865-879, 1983.

[10] S. Wagner, M. M. Klepsch, S. Schlegel et al., "Tuning Escherichia coli for membrane protein overexpression," Proceedings of the National Academy of Sciences of the United States of America, vol. 105, no. 38, pp. 14371-14376, 2008.

[11] W. C. W. Chan and S. Nie, "Quantum dot bioconjugates for ultrasensitive nonisotopic detection," Science, vol. 281, no. 5385, pp. 2016-2018, 1998.

[12] X. Michalet, F. Pinaud, T. D. Lacoste et al., "Properties of fluorescent semiconductor nanocrystals and their application to biological labeling," Single Molecules, vol. 2, no. 4, pp. 261-276, 2001.

[13] X. Michalet, F. F. Pinaud, L. A. Bentolila et al., "Quantum dots for live cells, in vivo imaging, and diagnostics," Science, vol. 307, no. 5709, pp. 538-544, 2005.

[14] D. R. Larson, W. R. Zipfel, R. M. Williams et al., "Water-soluble quantum dots for multiphoton fluorescence imaging in vivo," Science, vol. 300, no. 5624, pp. 1434-1436, 2003.

[15] M. Dahan, S. Lévi, C. Luccardini, P. Rostaing, B. Riveau, and A. Triller, "Diffusion dynamics of glycine receptors revealed by single-quantum dot tracking," Science, vol. 302, no. 5644, pp. 442-445, 2003.

[16] R. Edgar, A. Rokney, M. Feeney et al., "Bacteriophage infection is targeted to cellular poles," Molecular Microbiology, vol. 68, no. 5, pp. 1107-1116, 2008.

[17] A. Biebricher, W. Wende, C. Escudé, A. Pingoud, and P. Desbiolles, "Tracking of single quantum dot labeled EcoRV sliding along DNA manipulated by double optical tweezers," Biophysical Journal, vol. 96, no. 8, pp. L50-L52, 2009.

[18] Y. Ebenstein, N. Gassman, S. Kim et al., "Lighting up individual DNA binding proteins with quantum dots," Nano Letters, vol. 9, no. 4, pp. 1598-1603, 2009.

[19] L. Jauffred, A. C. Richardson, and L. B. Oddershede, "ThreeDimensional optical control of individual quantum dots," Nano Letters, vol. 8, no. 10, pp. 3376-3380, 2008.

[20] L. Jauffred and L. B. Oddershede, "Two-photon quantum dot excitation during optical trapping," Nano Letters, vol. 10, no. 5, pp. 1927-1930, 2010.

[21] S. Marín, S. Pujals, E. Giralt, and A. Merkoçi, "Electrochemical investigation of cellular uptake of quantum dots decorated with a proline-rich cell penetrating peptide," Bioconjugate Chemistry, vol. 22, no. 2, pp. 180-185, 2011.

[22] I. J. Finkelstein, M. L. Visnapuu, and E. C. Greene, "Singlemolecule imaging reveals mechanisms of protein disruption by a DNA translocase," Nature, vol. 468, no. 7326, pp. 983-987, 2010.

[23] R. T. Pomerantz, R. Ramjit, Z. Gueroui et al., "A tightly regulated molecular motor based upon T7 RNA polymerase," Nano Letters, vol. 5, no. 9, pp. 1698-1703, 2005.

[24] B. He, M. Rong, D. Lyakhov et al., "Rapid mutagenesis and purification of phage RNA polymerases," Protein Expression and Purification, vol. 9, no. 1, pp. 142-151, 1997.

[25] D. Beckett, E. Kovaleva, and P. J. Schatz, "A minimal peptide substrate in biotin holoenzyme synthetase-catalyzed biotinylation," Protein Science, vol. 8, no. 4, pp. 921-929, 1999.

[26] E. Gasteiger, C. Hoogland, A. Gattiker et al., The Proteomics Protocols Handbook: Protein Identification and Analysis Tools on the ExPASy Server, Humana Press, 2003. 
[27] J. Tholstrup, L. B. Oddershede, and M. A. Sørensen, "mRNA pseudoknot structures can act as ribosomal roadblocks," Nucleic Acids Research, vol. 40, pp. 303-313, 2012.

[28] D. Sage, F. R. Neumann, F. Hediger, S. M. Gasser, and M. Unser, "Automatic tracking of individual fluorescence particles: application to the study of chromosome dynamics," IEEE Transactions on Image Processing, vol. 14, no. 9, pp. 1372-1383, 2005.

[29] R. Edgar, M. McKinstry, J. Hwang et al., "High-sensitivity bacterial detection using biotin-tagged phage and quantum-dot nanocomplexes," Proceedings of the National Academy of Sciences of the United States of America, vol. 103, no. 13, pp. 48414845, 2006.

[30] W. Bücking, S. Massadeh, A. Merkulov, S. Xu, and T. Nann, "Electrophoretic properties of BSA-coated quantum dots," Analytical and Bioanalytical Chemistry, vol. 396, no. 3, pp. 1087-1094, 2010.

[31] S. J. Clarke, C. A. Hollmann, F. A. Aldaye, and J. L. Nadeau, "Effect of ligand density on the spectral, physical, and biological characteristics of CdSe/ZnS quantum dots," Bioconjugate Chemistry, vol. 19, no. 2, pp. 562-568, 2008.

[32] R. Mittal and M. P. Bruchez, "Biotin-4-fluorescein based fluorescence quenching assay for determination of biotin binding capacity of streptavidin conjugated quantum dots," Bioconjugate Chemistry, vol. 22, no. 3, pp. 362-368, 2011.

[33] H. Zhang, X. Zeng, Q. Li, M. Gaillard-Kelly, C. R. Wagner, and D. Yee, "Fluorescent tumour imaging of type I IGF receptor in vivo: comparison of antibody-conjugated quantum dots and small-molecule fluorophore," British Journal of Cancer, vol. 101, no. 1, pp. 71-79, 2009.

[34] C. M. Niemeyer, M. Adler, B. Pignataro et al., "Self-assembly of DNA-streptavidin nanostructures and their use as reagents in immuno-PCR," Nucleic Acids Research, vol. 27, no. 23, pp. 45534561, 1999.

[35] J. Richter, M. Adler, and C. M. Niemeyer, "Monte Carlo simulation of the assembly of bis-biotinylated DNA and streptavidin," ChemPhysChem, vol. 4, no. 1, pp. 79-83, 2003.

[36] L. Jauffred, M. Sletmoen, F. Czerwinski, and L. B. Oddershede, "Quantum dots as handles for optical manipulation," in Optical Trapping and Optical Micromanipulation VII, vol. 7762 of SPIE Proceedings, 2010.

[37] P. Thomen, P. J. Lopez, U. Bockelmann, J. Guillerez, M. Dreyfus, and F. Heslot, "T7 RNA polymerase studied by force measurements varying cofactor concentration," Biophysical Journal, vol. 95, no. 5, pp. 2423-2433, 2008.

[38] J. H. Kim and R. G. Larson, "Single-molecule analysis of 1D diffusion and transcription elongation of T7 RNA polymerase along individual stretched DNA molecules," Nucleic Acids Research, vol. 35, no. 11, pp. 3848-3858, 2007.

[39] G. M. Skinner, C. G. Baumann, D. M. Quinn, J. E. Molloy, and J. G. Hoggett, "Promoter binding, initiation, and elongation by bacteriophage T7 RNA polymerase: a single-molecule view of the transcription cycle," Journal of Biological Chemistry, vol. 279, no. 5, pp. 3239-3244, 2004.

[40] G. M. Skinner, B. S. Kalafut, and K. Visscher, "Downstream DNA tension regulates the stability of the T7 RNA polymerase initiation complex," Biophysical Journal, vol. 100, no. 4, pp. 10341041, 2011. 

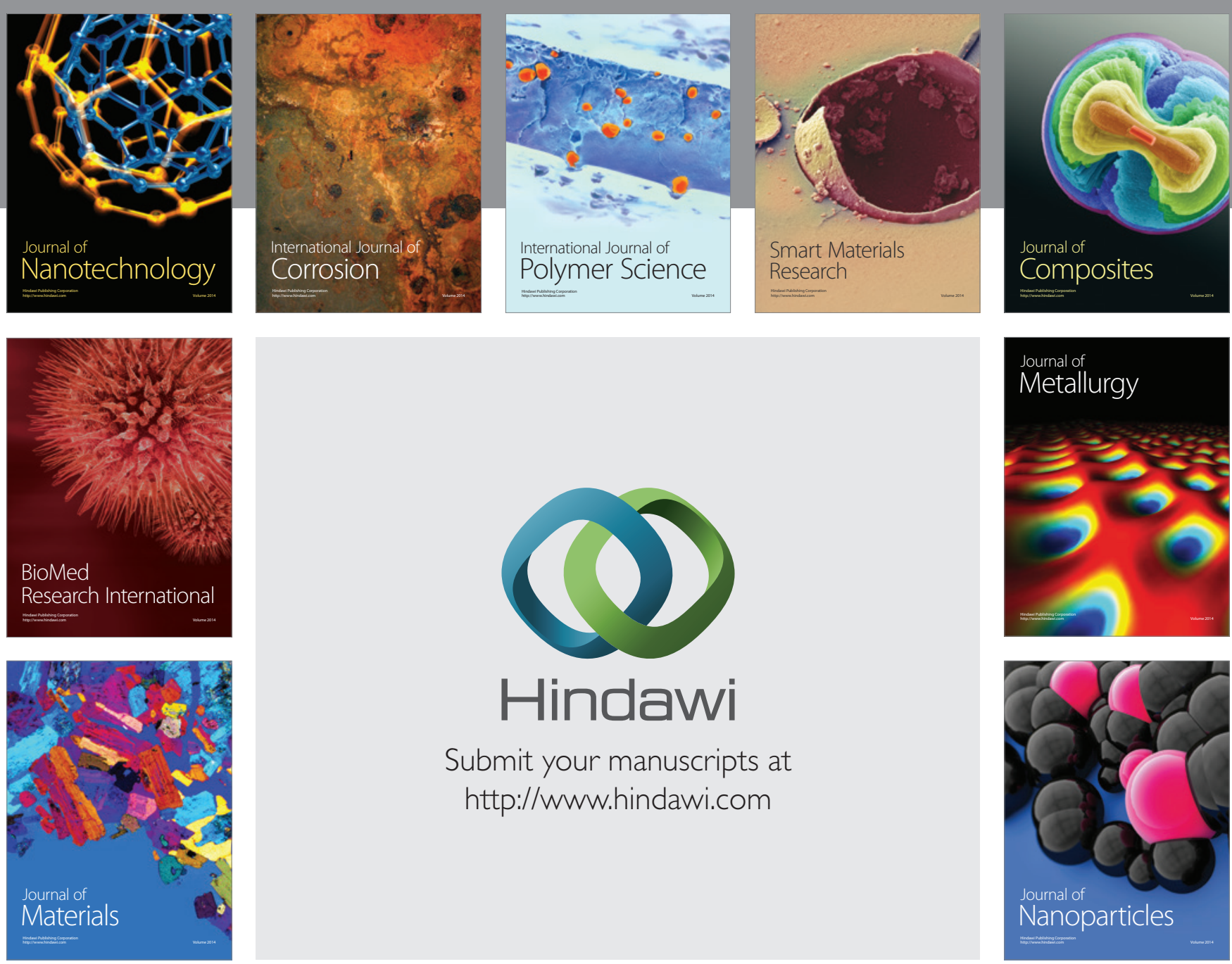

Submit your manuscripts at http://www.hindawi.com
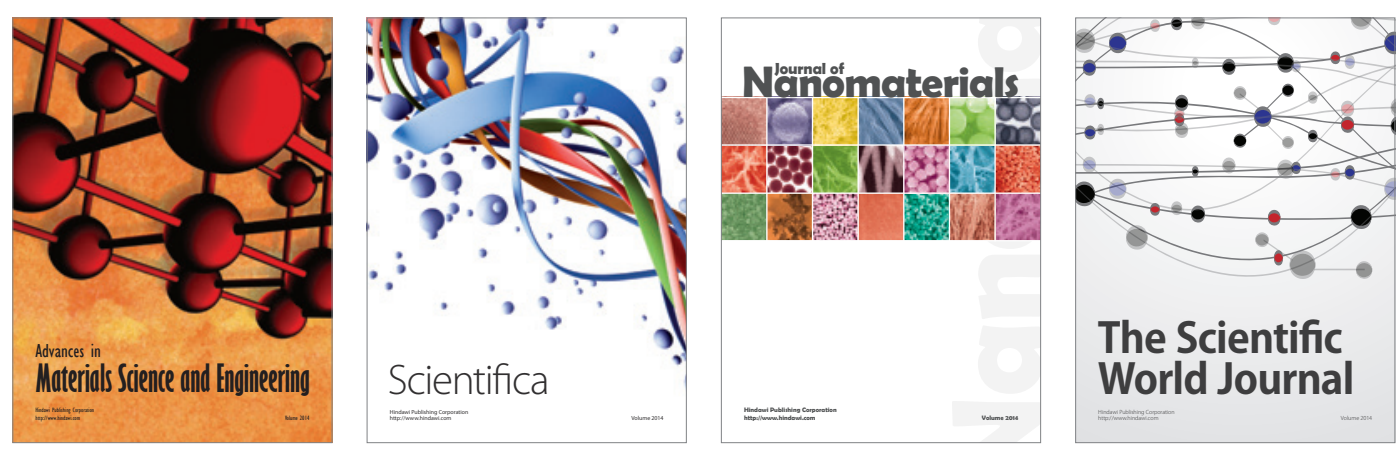

\section{The Scientific World Journal}
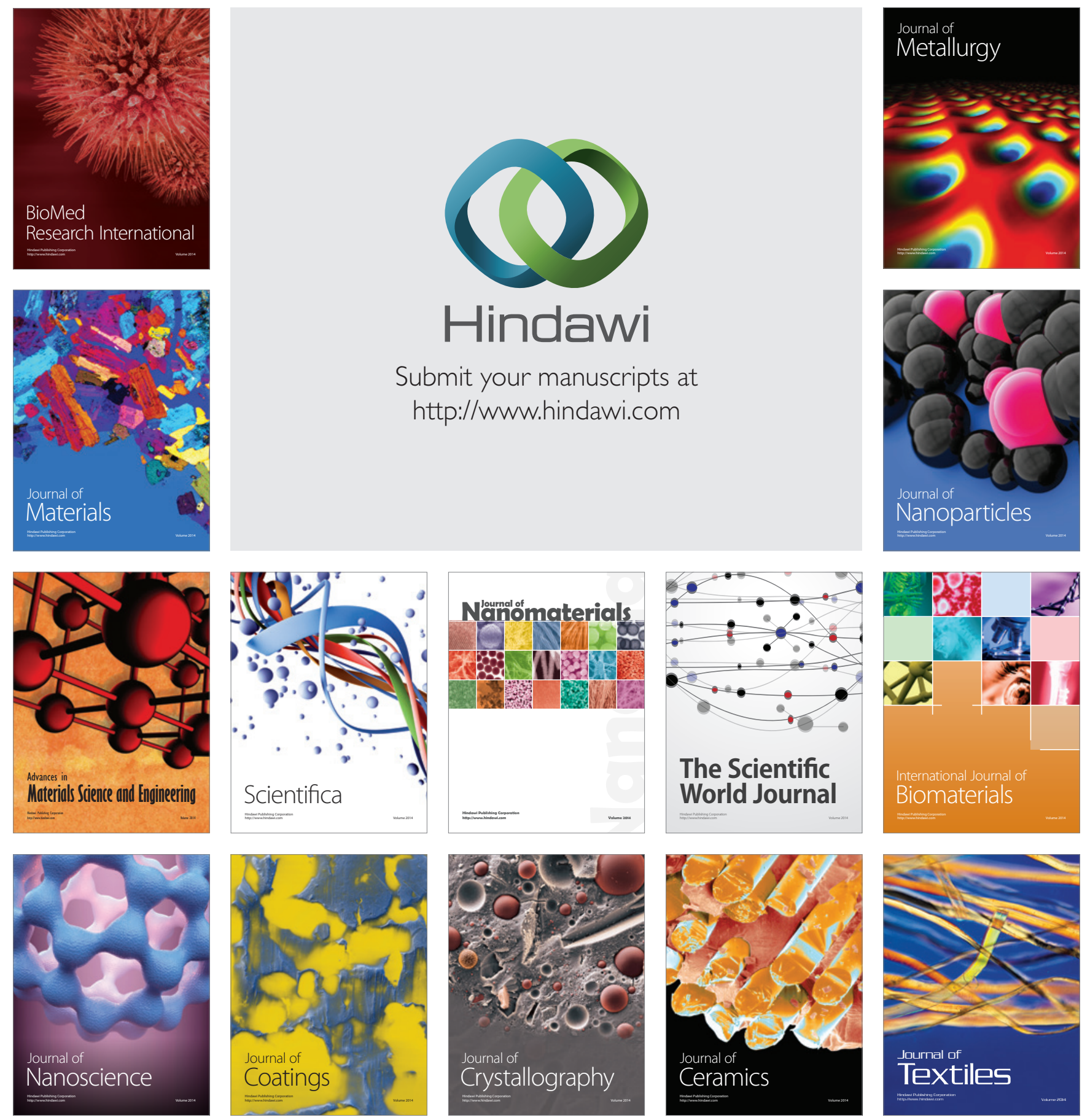